\title{
Les effets combinés du climat et des pressions anthropiques sur la forêt classée de Toéssin, Burkina Faso
}

\author{
Mamounata BELEM $^{1^{*}}$, Mathieu ZOUNGRANA ${ }^{2}$ et Moumouni NABALOUM ${ }^{3}$ \\ ${ }^{I}$ Département Environnement et Forets, INERA/CNRST, 03 BP 7047 Ouagadougou 03, Burkina Faso. \\ ${ }^{2}$ Unité de Formation et de Recherche en Sciences Humaines, Laboratoire d'Etudes et de Recherches sur les \\ Milieux et les Territoires, Université Ouaga I Prof Joseph KI-ZERBO, 76172186/79088188 ; \\ 03 BP: 7021 Ouagadougou, Burkina Faso. \\ ${ }^{3}$ Unité de Formation et de Recherche en Sciences Humaines, Laboratoire de Dynamique des Espaces et \\ des Sociétés, Université Ouaga I Prof Joseph KI-ZERBO, 60934423 ; \\ 03 BP: 7021 Ouagadougou 03, Burkina Faso. \\ *Auteur correspondant, E-mail : bmamounata@gmail.com, Tel : +22670 712977
}

\section{RÉSUMÉ}

Le Burkina Faso est confronté à de graves problèmes de déséquilibres écologiques et de dégradation accélérée des ressources naturelles. De nombreuses forêts classées sont, de ce fait, menacées de disparition dont les facteurs sont d'ordres climatique et anthropique. L'objectif de cette étude est d'analyser les incidences du climat et des actions anthropiques sur l'environnement de la forêt classée de Toéssin afin de proposer de meilleures actions de sa gestion. Pour ce faire, une recherche documentaire et des enquêtes ont été réalisées. Également, des images Landsat TM et ETM+ ont été traitées. Il ressort de cette étude que les pressions humaines qui pèsent sur cette aire protégée concernent principalement l'agriculture, l'élevage, la coupe frauduleuse du bois. Aussi note-t-on que les populations riveraines ne sont pas impliquées dans la gestion de la forêt ce qui entraîne davantage sa dégradation. Le massif forestier de Toéssin actuellement fragile a besoin d'être protégé et bien géré. Pour éviter son déclassement comme d'autres, plusieurs actions doivent être menées dont des opérations de reboisement, de déguerpissement des champs clandestins et d'implication de tous les utilisateurs des produits forestiers dans la gestion de la forêt.

(C) 2018 International Formulae Group. All rights reserved.

Mots clés: Burkina Faso, changement climatique, aire protégée, gestion forestière, produits forestiers.

\section{The combined effects of climate and anthropogenic pressures on the classified forest of Toéssin, Province of Passoré, Burkina Faso}

\begin{abstract}
Burkina Faso is facing serious ecological imbalances and accelerated deterioration of natural resources. As a result, many classified forests are endangered whose factors are anthropogenic and climate. The objective of this study is to analyze the impact of climate and human activities on the environment of the classified forest of Toessin in order to offer best actions of its management. To do this, a literature search and field investigations were conducted. Also, Landsat TM images and ETM + have been processed. It is apparent from
\end{abstract}


this study that human pressures that affect this protected area are mainly agriculture, husbandry, the fraudulent wood cutting. We also noted that residents are not involved in the management of the forest resulting in more degradation. The currently fragile Toessin forest has needs to be protected and well managed. To avoid its decommissioning as other forests, several actions must be taken. It is necessary to organize periodically reforestation operations, run the clandestine fields and involve all users of forest products in the forest management.

(C) 2018 International Formulae Group. All rights reserved.

Keywords: Burkina Faso, climate change, protected area, forest management, forest products.

\section{INTRODUCTION}

Le Burkina Faso dispose de plusieurs aires protégées dont certaines sont érigées en forêts classées et réserves internationales. Elles représentent un immense réservoir de ressources biologiques dont dépendent fortement les populations riveraines. Les objectifs qui leur sont assignés sont nombreux. Il ressort de cette catégorisation que la conservation et la gestion durable de la biodiversité au profit des générations futures constitue une préoccupation nationale (Doamba, 2012).

Depuis 1993, la région sahélienne fait face à un nouveau type de variabilité interannuelle de la pluviométrie caractérisée par une alternance brutale entre années humides et années très sèches. Les changements enregistrés après les années 1970 sont plus significatifs par rapport à la référence 1940-1969 (Abdou, 2010). Le Burkina Faso n'a pas été épargné de ces nombreuses crises climatiques. On constate une dégradation progressive des conditions climatiques qui se manifestent sous différentes formes: les sécheresses dues à l'insuffisance des pluies et leur mauvaise répartition spatiotemporelle, les inondations provenant des pluies diluviennes, les pics de chaleur, les vents violents et les vents de sable. Ces phénomènes qui sont de plus en plus réguliers confirment la réalité du changement climatique et ses impacts sur tous les secteurs de développement. Ces crises climatiques ont des répercussions graves sur l'environnement. Les aires protégées et leurs écosystèmes deviennent vulnérables face aux changements des régimes des précipitations, à l'augmentation de la température, etc.
En outre, sous la pression de la croissance démographique conjuguée à une paupérisation grandissante, les aires protégées sont de plus en plus menacées. $\mathrm{La}$ conséquence de la croissance démographique contraint les paysans à rechercher de nouvelles terres agricoles au détriment des milieux naturels et entraîne également une consommation plus importante des ressources (Curtis, 2014 ; MECV, 2004). Selon Tarchiani et al. (2008), au Burkina Faso, sur un total de soixante-huit forêts classées, quatre seulement ne représentent pas de signe d'occupation ou d'exploitation. La plupart des forêts classées connaissent une dégradation dont l'ampleur varie d'une localité à une autre. Faute de moyens (humains, financiers et matériel) et d'intérêt marqué, elles sont les moins entretenues comparativement aux aires fauniques et presque toutes sont soumises aux pressions agricoles, pastorales et aux feux de brousse. Certaines forêts classées doivent leur existence que par leur nom (Belemsobgo et al., 2006). Au regard de ces constats, nous nous sommes intéressés à la Forêt classée de Toéssin. L'objectif principal de cette étude est d'analyser les incidences du climat et des actions anthropiques sur l'environnement de la forêt afin de proposer de meilleures actions de sa gestion. Il s'agit spécifiquement : i) de montrer l'ampleur de la dégradation de la forêt; ii) d'analyser les impacts des paramètres climatiques (pluviométrie et température) et de l'action de l'homme sur les ressources naturelles de la forêt; iii) de mesurer le niveau d'implication des populations locales dans la gestion de la forêt. 


\section{MATERIEL ET METHODES}

\section{Le site de l'étude}

L'analyse a concerné la zone de la forêt classée de Toéssin et les villages riverains. La forêt est située dans la commune rurale de Samba dans la province du Passoré, à environ $30 \mathrm{~km}$ au Sud-ouest de la ville de Yako sur l'axe routier Yako-Koudougou (RN13). Le classement de la forêt est intervenu le 24 juin 1954 par arrêté de classement $\mathrm{N}^{\circ} 4838 / \mathrm{SEF}$ et couvrait une superficie de 490 ha. Elle porte le nom du village de Toéssin qui le jouxte. La superficie de la forêt a été agrandie à 774 ha en 1991. L'agrandissement a concerné la partie Nord et Est des anciennes limites. Cependant, les nouvelles limites de la forêt ne sont pas officiellement reconnues. De nombreux auteurs se réfèrent toujours à la superficie de l'arrêté de 1954. Elle est limitée au Nord par le village de Minissia, à l'Est par le village de Itian, au Sud par le village de Toéssin et à l'Ouest par le village de Mesga et la RN13 (Figure 1).

\section{Méthode de collecte}

Dans le cadre de cette étude, plusieurs catégories de données ont été collectées. Il s'agit des documents, des données collectées auprès des populations, des données climatiques et des données spatiales. La recherche bibliographique a permis d'avoir des connaissances sur la technique du traitement d'images, les principaux facteurs de dégradation et de gestion des forêts. Pour les enquêtes, un questionnaire a été administré de façon aléatoire aux populations de deux villages riverains de la forêt, Toéssin et Mesga. Au total, 57 personnes dont l'âge est compris entre 25 et 80 ans ont été interrogées sur ces deux villages soit $65 \%$ à Toéssin et $35 \%$ à Mesga. Un guide d'entretien a été également adressé à l'agent forestier en poste dans la commune de Samba. Elles ont fourni des informations sur la perception de l'évolution de la pluviométrie et la température de la zone, les potentialités des ressources forestières, l'évolution et la gestion de la forêt. En outre, des données climatiques d'une série de 30 ans (1983-2012), obtenues auprès de la Direction de la Météorologie Nationale ont été utilisées pour étudier l'évolution de la pluviométrie et de température. Enfin, un matériel adapté a servi pour la réalisation de la cartographie de l'occupation des terres entre 1986 et 2010. Trois types de données spatiales ont été utilisés : le premier concerne les données issues de la Base nationale de données topographiques (BNDT) produite par l'Institut Géographique du Burkina (IGB). Elle contient des couches des limites des aires protégées. Le second est constitué de trois images satellitales multidates de types Landsat : deux images TM 1986 et 1999, et une image ETM+ 2010. Ces images ont été prises entre le mois d'octobre et de novembre. À ces données, s'ajoutent celles collectées sur le terrain au moyen de GPS. Les relevés GPS sont indispensables pour la classification des images.

\section{Traitement d'images et cartographie}

Le traitement a commencé par la correction géométrique des images avec le logiciel Erdas Imagine 9.2. On a procédé à une registration afin de corriger et géoréferencer les images. La correction des effets atmosphérique a été possible grâce à un calibrage des images. Cette action permet de transformer les comptes numériques en réflectance apparente. La combinaison 5/4/3 (moyen infrarouge, proche infrarouge, rouge) a été retenue pour la combinaison colorée, elle offre plus de contraste entre les différentes unités d'occupation des terres (Bagaya, 2013). Des unités d'occupations identifiées sur le terrain ont guidé le choix des sites d'entrainement lors de la classification. Il s'agit d'une classification supervisée de l'image avec l'algorithme du maximum de vraisemblance. Pour l'évaluation de la qualité de la classification, il faut utiliser, la matrice de confusion qui est un tableau permettant d'évaluer la fiabilité de la classification. En effet, pour une précision supérieure à $60 \%$, la classification est acceptable (Sawadogo, 2013). Les précisions de classification des trois images $(1986,1999,2010)$ sont respectivement $91,971 \%, 94,97 \%$ et $92,3 \%$. Dans l'ensemble, la classification des images est satisfaisante même s'il y a des erreurs d'affectation des pixels entre certaines classes. Les images traitées sont transférées sur le logiciel ArcGis 10 pour la vectorisation, l'analyse et la production cartographique proprement dit. 


\section{Analyse statistique}

La matrice de transition est un tableau à double entrées (Tableau 1) qui permet de décrire de manière condensée, les changements d'état des cellules d'occupation des terres entre deux dates données (Schlapfer., 2002 cité par Oloukoi et al., 2006).

Dans la matrice de transition, le nombre de lignes de la matrice représente le nombre d'unités d'occupation des terres $t_{0}$ qui est la date de départ. Le nombre de colonnes quant à lui, représente le nombre d'unités d'occupation à la période $t_{1}$. La case $a(i, j) d e$ la matrice correspond à la superficie d'une catégorie i d'occupation des terres au temps $\mathrm{t}_{0}$, converti à une catégorie $\mathrm{j}$ au temps $\mathrm{t}_{1}$. Les changements se font donc de la ligne i vers la colonne $\mathrm{j}$.
La diagonale de la matrice contient la superficie des zones qui sont restées inchangées entre les périodes $t_{0}$ et $t_{1}$. La somme $\mathrm{Eit}_{0}=\sum \mathrm{a}(\mathrm{i}, \mathrm{j})$ de la ligne $\mathrm{i}$ correspond à la surface totale de l'unité i d'occupation des terres au temps $t_{0}$, de même, la somme $\mathrm{Ejt}_{1}=\sum \mathrm{a}(\mathrm{i}, \mathrm{j})$ de la colonne $\mathrm{j}$ constitue la superficie totale de l'unité $\mathrm{j}$ d'occupation des terres au moment $t_{1}$ (Oloukoi et al., 2006).

Dans le cadre de la présente étude, le calcul de la superficie ayant transité, a été faite à partir de l'union des deux couches d'occupation des terres concernées. Ensuite, nous avons procédé au calcul de la superficie de cette union avant de l'exporter sur Excel pour la réalisation de la matrice de transition à l'aide d'un tableau dynamique croisé entre les deux dates concernées.

Tableau 1 : Matrice de transition entre les dates t0 et $\mathrm{t} 1$.

\begin{tabular}{lcccc}
\hline $\begin{array}{l}\text { Unités d'occupation des } \\
\text { terres } \mathbf{i} \text { au temps } \mathbf{t}_{\mathbf{0}}\end{array}$ & \multicolumn{4}{c}{ Unités d'occupation des terres $\mathbf{j}$ au temps $\mathbf{t}_{\mathbf{1}}$} \\
\cline { 2 - 5 } & Unité $\mathbf{1}(\mathbf{j}=\mathbf{1})$ & Unité $\mathbf{2}(\mathbf{j}=\mathbf{2})$ & Unité $\mathbf{3}(\mathbf{j}=\mathbf{3})$ & Sommes Eit $\mathbf{d e s}_{\mathbf{0}}$ lignes \\
\hline Unité $1(\mathrm{i}=1)$ & $\mathrm{a}(1,1)$ & $\mathrm{a}(1,2)$ & $\mathrm{a}(1,3)$ & $\mathrm{E}_{1} \mathrm{t}_{0}=\sum \mathrm{a}(1, \mathrm{j})$ \\
Unité $2(\mathrm{i}=2)$ & $\mathrm{a}(2,1)$ & $\mathrm{a}(2,2)$ & $\mathrm{a}(2,3)$ & $\mathrm{E}_{2} \mathrm{t}_{0}=\sum \mathrm{a}(2, \mathrm{j})$ \\
Unité $3(\mathrm{i}=3)$ & $\mathrm{a}(3,1)$ & $\mathrm{a}(3,2)$ & $\mathrm{a}(3,3)$ & $\mathrm{E}_{3} \mathrm{t}_{0}=\sum \mathrm{a}(3, \mathrm{j})$ \\
Sommes Ejt $\mathrm{j}_{1}$ des colonnes & $\mathrm{E}_{1} \mathrm{t}_{1}=\sum \mathrm{a}(\mathrm{i}, 1)$ & $\mathrm{E}_{2} \mathrm{t}_{1}=\sum \mathrm{a}(\mathrm{i}, 2)$ & $\mathrm{E}_{3} \mathrm{t}_{1}=\sum \mathrm{a}(\mathrm{i}, 3)$ & $\sum \sum \mathrm{a}(\mathrm{i}, \mathrm{j})$ \\
\hline
\end{tabular}

Oloukoi et al., 2006.

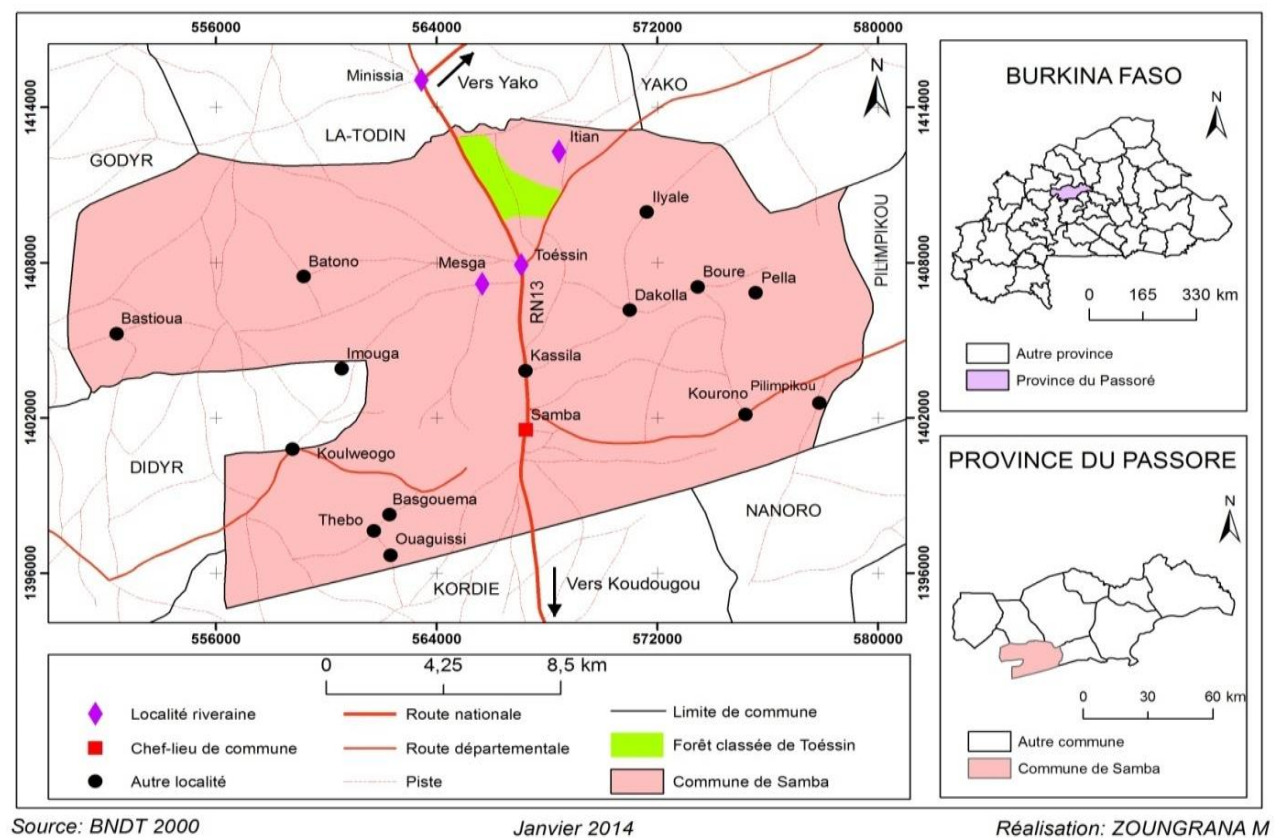

Figure 1 : Carte de localisation de la forêt classée de Toéssin. 


\section{RÉSULTATS \\ Évolution de l'occupation des terres De 1986 à 1999}

La Figure 2 et le Tableau 2 permettent de faire les constats suivants sur l'évolution de l'occupation des terres entre 1986 et 1999 de la forêt.

- 46,339 ha de la végétation dense sont restés dans cette même d'unité d'occupation des terres en 1999, tandis que 145, 364 ha soit $75,82 \%$ de la superficie de cette unité a été converti en d'autres catégories. Une grande proportion de la savane arborée soit 71,59\% de la superficie de cette unité a été transformée en savane arbustive.

- 263,629 ha de la végétation claire sont demeurés dans cette même catégorie d'occupation des terres en 1999, alors que 46,869 ha soit $15,09 \%$ de la superficie de cette catégorie a été converti en d'autres unités. En effet, 3,74\% s'est converti en champs, 5,13\% en zone nue et $6,22 \%$ en savane arborée.

\section{De 1999 à 2010}

La Figure 2 et le Tableau 2 permettent de faire les constats suivants sur l'évolution de l'occupation des terres entre 1999 et 2010 de la forêt.

- 19,023 ha de la végétation dense sont restés inchangés en 2010, alors que 46,705 ha soit $71,06 \%$ a été transformé en d'autres unités d'occupation. 5,975 ha de la superficie totale de la savane arborée soit $9,09 \%$ a été convertis en champs et en zone nue.

- 308,062 ha de végétation claire sont demeurés dans cette même d'unité d'occupation des terres en 2010, tandis que 120,396 ha soit $28,06 \%$ a été convertis en d'autres catégories d'occupation. 19,94\% de la superficie totale de la savane arbustive s'est transformée en champs et $6,47 \%$ en zone nue.

- Des portions des unités d'occupation des terres telles que les champs, les zones nues, la savane arborée et arbustive sont convertis en plan d'eau d'une superficie totale de 0,825 ha.

\section{Analyse de l'évolution des unités d'occupation des terres}

Les tableaux 3 et 4 , quant à eux permettent d'apprécier la matrice de transition des unités d'occupation des terres entre 1986 et 1999 et de 1999 à 2010.

La Figure 3 donne les unités d'occupation des terres en 1986, 1999 et 2010. On constate comme suit, que les différentes unités d'occupation de la forêt ont beaucoup évolué entre 1986 et 2010.

\section{La végétation dense}

La superficie de la savane arborée qui était de 191,703 ha en 1986 est passée à 65,728 ha en 1999 , soit une régression de $65,71 \%$. En 2010 , la surface de la savane a connu une régression de 30,235 ha soit une perte de $46 \%$.

\section{La végétation claire}

En 1986, la superficie de la savane arbustive estimée à 310,498 ha était la principale unité d'occupation de la forêt $(55,73 \%)$. En 1999, on note une augmentation de la superficie de cette unité puisqu'elle est passée à 429,016 ha, soit un accroissement de $21,27 \%$. En revanche en 2010, on constate une régression de la superficie de la savane arbustive. Elle est passée à 357,652 ha, soit un recul de 71,364 ha.

\section{Les champs}

La superficie des champs qui était de 29,904 ha en 1986 a connu une légère diminution en 1999 puisqu'elle était évaluée à 28,373 ha, soit un recul de $0,27 \%$. Par contre en 2010, la superficie occupée par les champs a beaucoup évolué. Elle s'est quadruplée, car elle s'élève à 120,54 ha.

\section{Les zones nues}

La superficie des zones nues évolue de façon progressive dans la forêt classée. De 25,015 ha en 1986, elle est passée à 34,003 ha en 1999, soit une augmentation de 8,988 ha. En 2010, les zones nues occupaient une surface de 39,357 ha, soit une nouvelle hausse de 5,354 ha. En 24 ans, 14,342 ha ont été transformés en zones nues.

\section{Le plan d'eau}

Le traitement des images Landsat de 1986 et de 1999 révèle que le plan d'eau était inexistant. Sa superficie est estimée à 0,825 ha en 2010. 
Évolution des paramètres climatiques sur la période 1983-2012

La pluviométrie

La Figure 4 donne l'évolution interannuelle des précipitations de 1983 à 2012. L'examen de ce graphique permet de distinguer des années sèches et des années avec des pluviométries excédentaires. Les années 1983, 1984, 1985, 1987 et 1990 sont considérées comme des années sèches puisque les hauteurs d'eau enregistrées durant ces années sont largement inférieures (moins de $500 \mathrm{~mm} / \mathrm{an}$ ) à la moyenne de la série qui est de 647,61 mm. Cependant, au cours des années 1994, 2007, 2010 et 2012, la pluviométrie a été excédentaire (plus de 800 $\mathrm{mm} / \mathrm{an}$ ). Durant les dix dernières années (2003-2012), on constate une hausse globale de pluviométrie annuelle. La moyenne est de $741,37 \mathrm{~mm} / \mathrm{an}$. C'est ce qui explique l'évolution ascendante de la courbe de tendance. L'augmentation générale de la pluviométrie favorise le développement de la végétation.

\section{La température}

La Figure 5 indique la variation des températures interannuelles entre 1983 et 2012. Durant ces trois décennies, les températures moyennes annuelles ont fluctué de façon significative. L'année 1994 a été la moins chaude $\left(28,07^{\circ} \mathrm{C}\right)$ et l'année 2010 la plus chaude $\left(30,22^{\circ} \mathrm{C}\right)$. On constate aussi que la dernière décennie a été la plus chaude par rapport aux deux premières. La température moyenne décennale est de $28,84^{\circ} \mathrm{C}$ entre 1983-1992, $28,82{ }^{\circ} \mathrm{C}$ entre $1993-2002$ et 29,64 entre 2003-2012. Le résultat de l'analyse des données de la série corrobore avec la perception des populations riveraines de la forêt. La tendance générale des températures est à la hausse.

\section{L'évapotranspiration (ETP)e}

La Figure 6 révèle l'évolution de l'évapotranspiration de 1983 à 2012. Il s'agit de l'évolution de la moyenne mensuelle de la quantité d'eau totale transférée du sol vers l'atmosphère (évapotranspiration). Au cours de l'année, la variation de l'ETP est influencée par l'évolution de la pluviométrie et celle de la température. L'ETP augmente progressivement de janvier à mai. Les fortes valeurs sont observées durant cette période avec une maximale en mai $(212,50 \mathrm{~mm})$. Elle commence à diminuer à partir de juin pour se maintenir faiblement en août $(147,60 \mathrm{~mm})$. L'ETP augmente à nouveau entre septembre et octobre. On constate une certaine stabilité entre novembre $(166,62 \mathrm{~mm})$ et décembre $(166,84 \mathrm{~mm})$. Les valeurs de l'ETP les plus importantes sont observées pendant les périodes enregistrant de faibles hauteurs d'eau et les périodes les plus chaudes. La forte évapotranspiration réduit l'humidité du sol.

Tableau 2 : Occupation des terres en 1986, 1999 et 2010.

\begin{tabular}{|c|c|c|c|c|c|c|}
\hline \multirow[t]{2}{*}{ Unités d'occupation } & \multicolumn{2}{|l|}{1986} & \multicolumn{2}{|l|}{1999} & \multicolumn{2}{|l|}{2010} \\
\hline & Superficie (ha) & $\%$ & Superficie (ha) & $\%$ & Superficie (ha) & $\%$ \\
\hline Végétation dense & 191,703 & 34,41 & 65,728 & 11,8 & 35,493 & 6,371 \\
\hline Végétation claire & 310,498 & 55,732 & 429,016 & 77,01 & 357,652 & 64,2 \\
\hline Champ & 29,904 & 5,368 & 28,373 & 5,093 & 123,793 & 22,22 \\
\hline Zone nue & 25,015 & 4,49 & 34,003 & 6,103 & 39,357 & 7,064 \\
\hline Plan d'eau & 0 & 0 & 0 & 0 & 0,825 & 0,149 \\
\hline Total & 557,12 & 100 & 557,12 & 100 & 557,12 & 100 \\
\hline
\end{tabular}




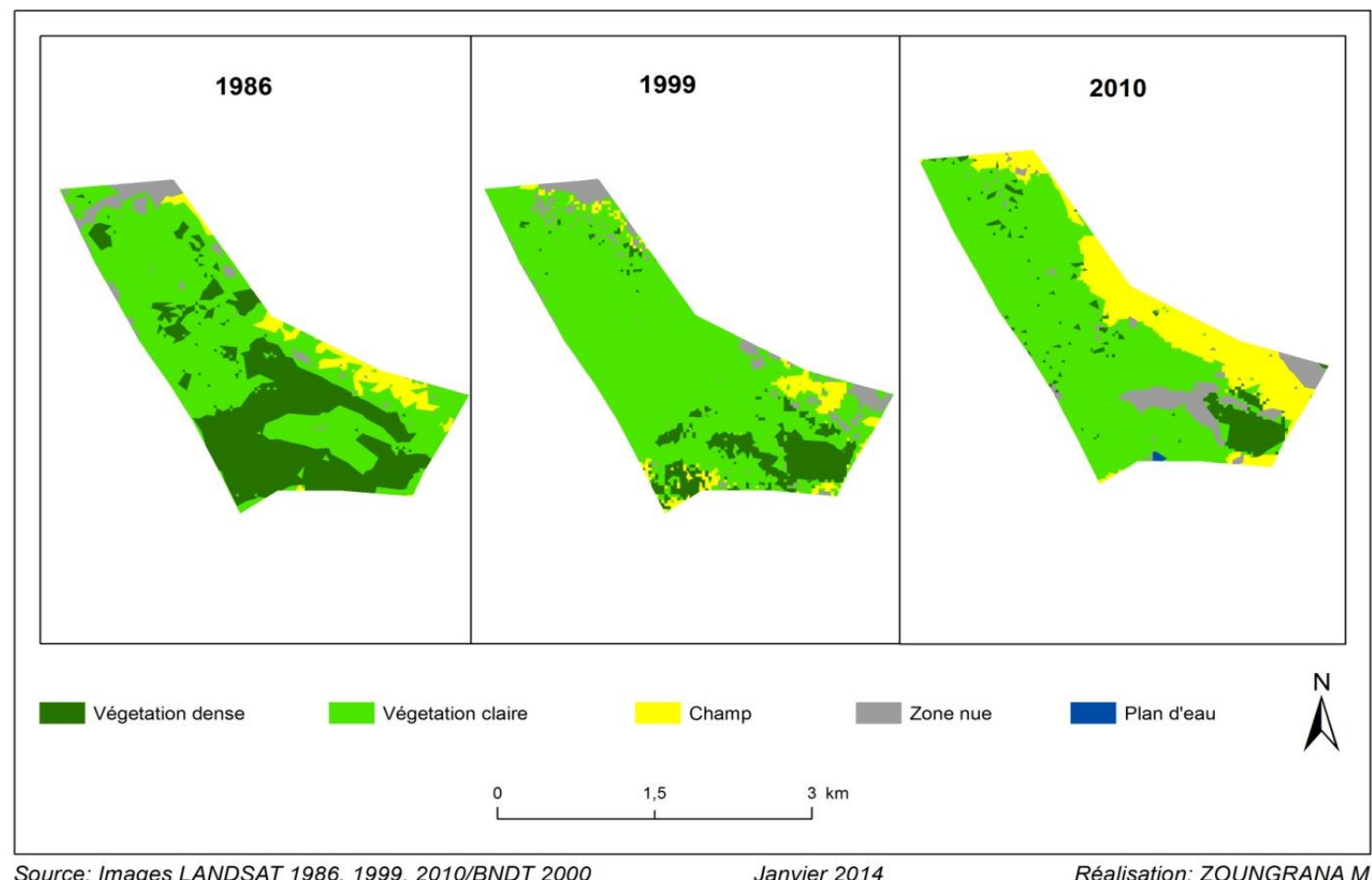

Figure 2 : Occupation des terres entre 1986, 1999 et 2010 de la forêt classée de Toéssin.

Tableau 3: Matrice de transition des unités d'occupation des terres entre 1986 et 1999.

\begin{tabular}{lccccc}
\hline & \multicolumn{5}{c}{ Unités d'occupation des terres en 1999 } \\
\hline $\begin{array}{l}\text { Unités d'occupation } \\
\text { des terres en 1986 }\end{array}$ & Champ & Zone nue & $\begin{array}{c}\text { Végétation } \\
\text { claire }\end{array}$ & $\begin{array}{c}\text { Végétation } \\
\text { dense }\end{array}$ & Total \\
\hline Champ & 9,686 & 5,710 & 14,467 & 0,041 & 29,904 \\
Zone nue & 0,887 & $\mathbf{1 0 , 4 2 4}$ & 13,670 & 0,034 & 25,015 \\
Végétation claire & 11,617 & 15,938 & $\mathbf{2 6 3 , 6 2 9}$ & 19,314 & 310,498 \\
Végétation dense & 6,183 & 1,931 & 137,250 & $\mathbf{4 6 , 3 3 9}$ & 191,703 \\
\hline Total & 28,373 & 34,003 & 429,016 & 65,728 & $\mathbf{5 5 7 , 1 2}$ \\
\hline
\end{tabular}

Source : traitement d'image Landsat TM 1986 et 1999

Tableau 4 : Matrice de transition des unités d'occupation des terres entre 1999 et 2010.

\begin{tabular}{lllllll}
\hline $\begin{array}{l}\text { Unités d'occupation } \\
\text { des terres en 1999 }\end{array}$ & \multicolumn{7}{c}{ Unités d'occupation des terres en 2010 } \\
\cline { 2 - 7 } & Champ & Zone nue & Végétation claire & $\begin{array}{l}\text { Végétation } \\
\text { Dense }\end{array}$ & $\begin{array}{c}\text { Plan } \\
\text { d'eau }\end{array}$ & Total \\
\hline Champ & $\mathbf{1 7 , 7 7 1}$ & 0,519 & 3,432 & 6,567 & 0,084 & 28,373 \\
Zone nue & 18,418 & $\mathbf{7 , 0 6 5}$ & 5,816 & 2,449 & 0,255 & 34,003 \\
Végétation claire & 85,656 & 27,746 & $\mathbf{3 0 8 , 0 6 2}$ & 7,454 & 0,098 & 429,016 \\
Végétation dense & 1,948 & 4,027 & 40,342 & $\mathbf{1 9 , 0 2 3}$ & 0,388 & 65,728 \\
\hline Total & 123,793 & 39,357 & 357,652 & 35,493 & $\mathbf{0 , 8 2 5}$ & $\mathbf{5 5 7 , 1 2}$ \\
\hline Source: traitement d'image Landsat TM 1999 et 2010 & & & &
\end{tabular}




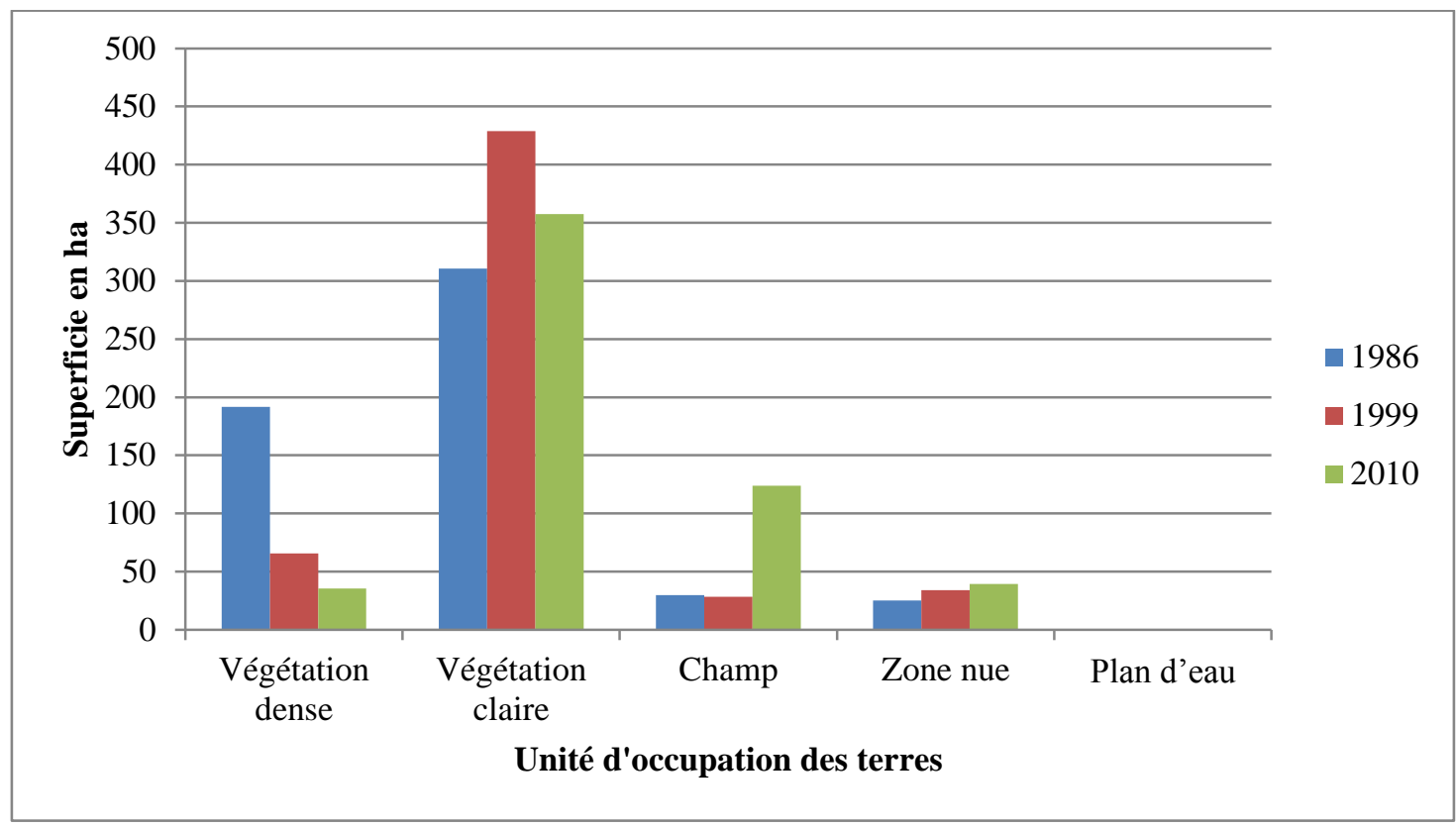

Source: Traitement des images Landsat 1986, 1999, 2010.

Figure 3 : Évolution des unités d'occupation des terres en 1986, 1999 et 2010.

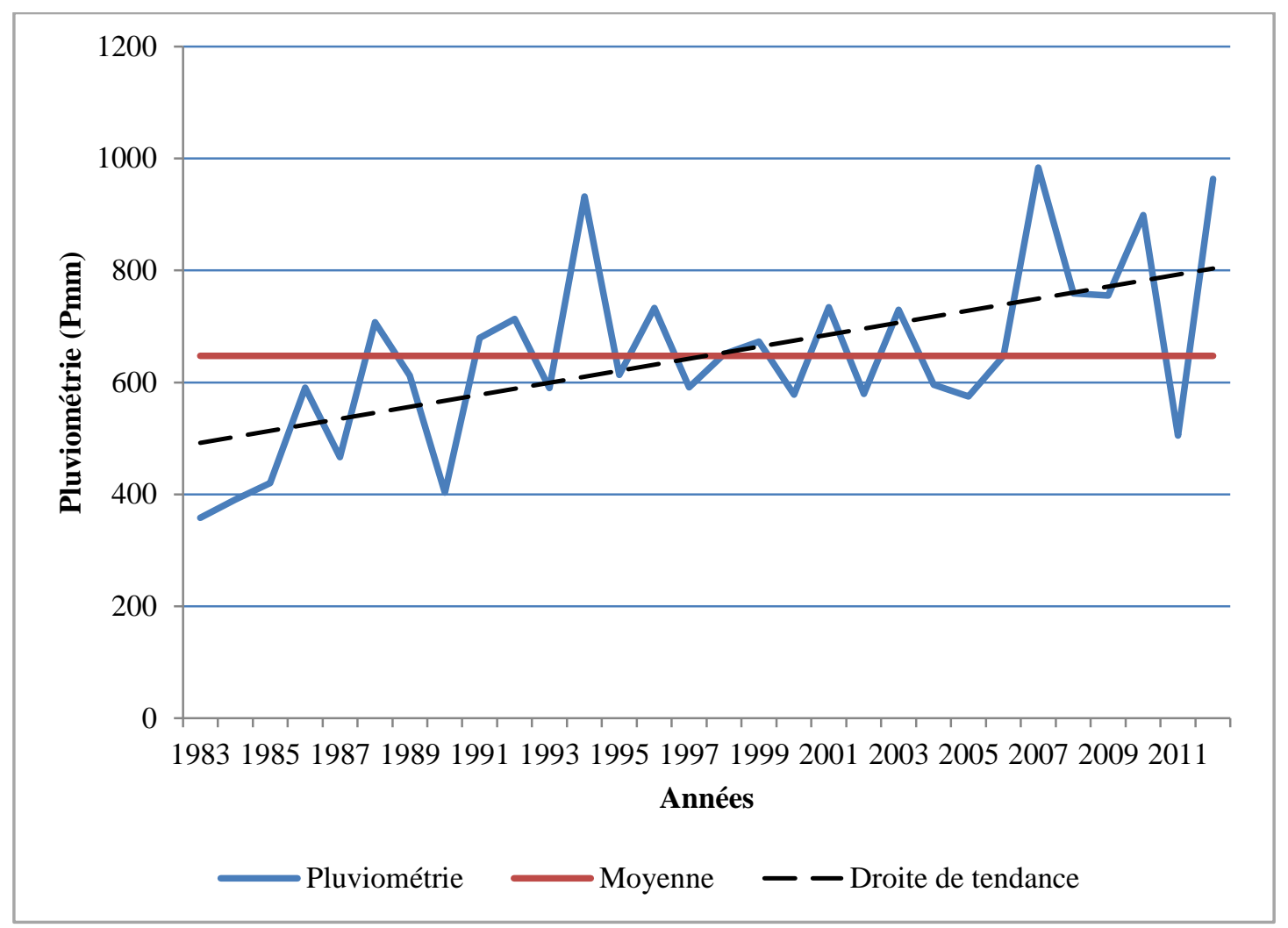

Source: Direction de la Météorologie Nationale du Burkina Faso.

Figure 4 : Évolution des précipitations de 1983 à 2012. 


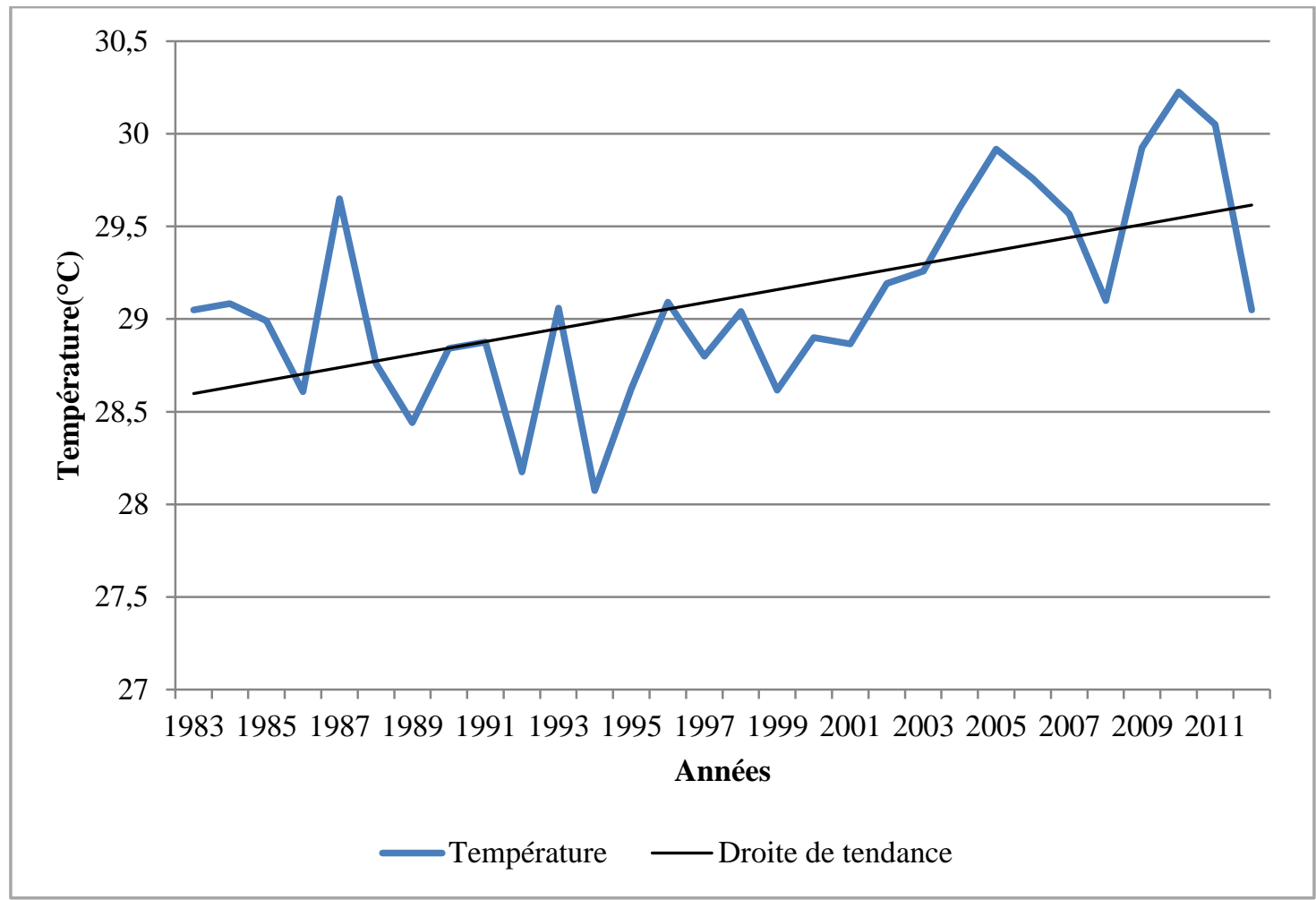

Source : Direction de la Météorologie Nationale du Burkina Faso.

Figure 5 : Évolution des températures moyennes de 1983 à 2012.

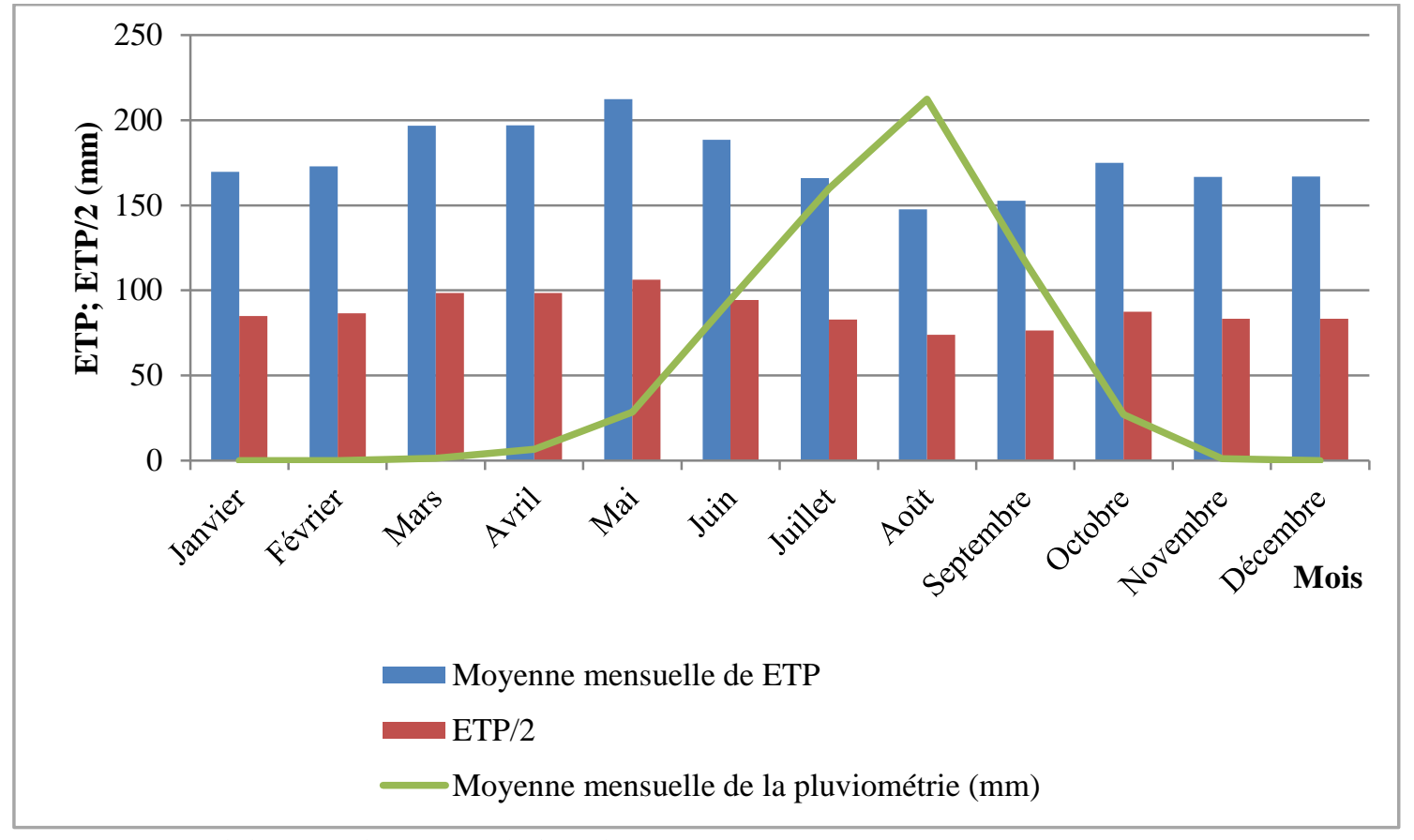

Source : Direction de la Météorologie Nationale du Burkina Faso.

Figure 6: Évolution de l'évapotranspiration de 1983 à 2012. 


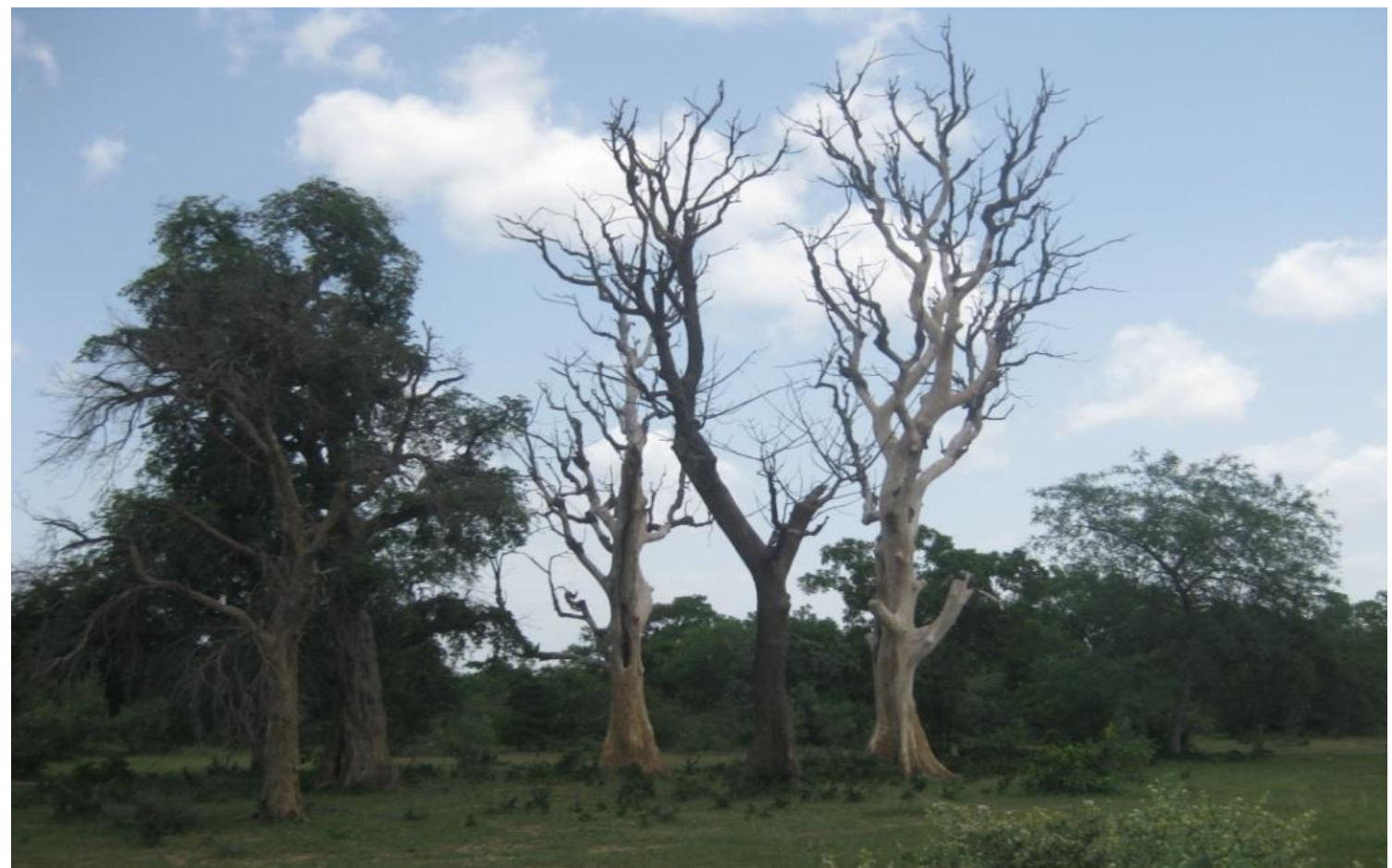

Source: Enquête de terrain, septembre 2013.

Figure 7: Des pieds d'arbres morts dans la forêt classée de Toéssin.

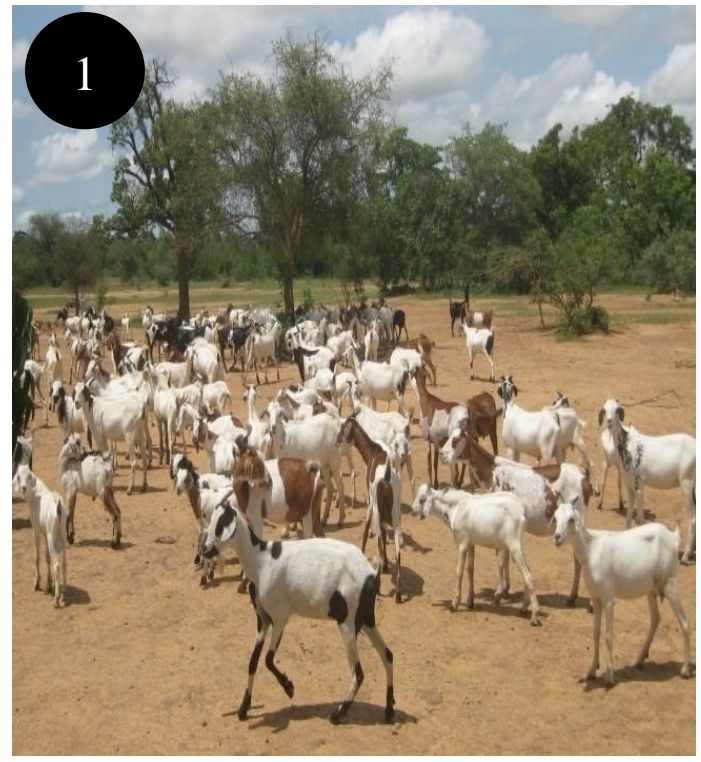

Des Ovins et Caprins

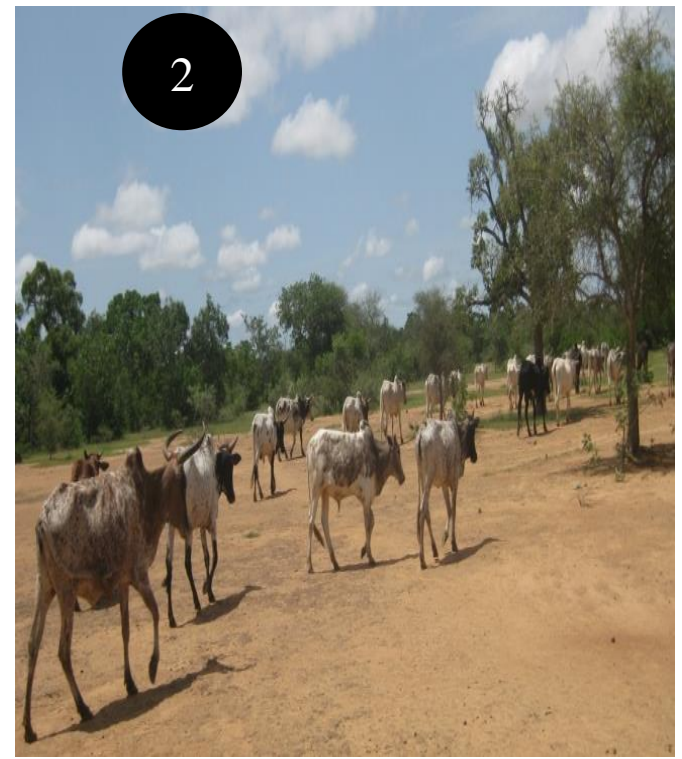

Des Bovins

Source: Enquête de terrain, septembre 2013.

Figures 8 et 9: Des animaux errant dans la forêt classée de Toéssin de gauche à droite. 

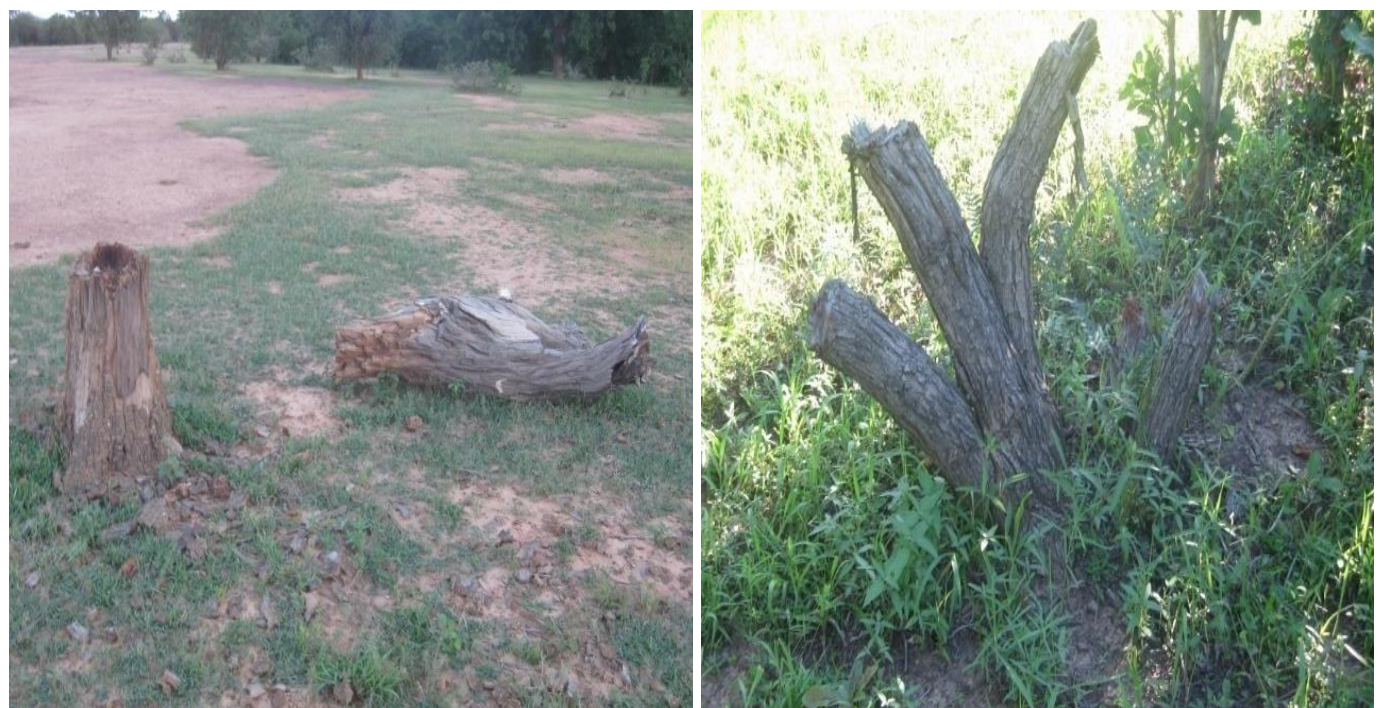

Source : Enquête de terrain, septembre 2013

Figures 10 et 11: Des restes d'arbres coupés dans la forêt classée de Toéssin de gauche à droite.

\section{DISCUSSION}

\section{Les impacts du changement climatique sur} les ressources forestières

\section{L'exploitation des produits forestiers}

Dans les pays en développement, les systèmes de production sont caractérisés par une agriculture de subsistance et un élevage à vocation agricole. A cause de la variabilité climatique et des risques climatiques croissants dans ces pays, les produits issus des forêts, comme le charbon, le bois de chauffage et les produits forestiers non ligneux constituent d'importants filets de sécurité et font partie des stratégies de diversification des revenus pour de nombreuses communautés (CIFOR, 2012, Guiguindibaye et al., 2013). Ces biens et services, fournis par les écosystèmes forestiers, constituent un moyen d'adaptation aux effets néfastes des périodes de mauvaises récoltes dues à une pluviométrie capricieuse. Selon Diatta et al. (2016), au Sénégal Cordyla pinnata est l'espèce la plus appréciée par les populations locales pour les revenus qu'elle procure par la vente de ses sous-produits.

Les mauvaises récoltes qu'enregistrent les paysans riverains de la forêt classée de Toéssin, accentuent l'exploitation des produits forestiers. Les populations qui n'ont pas d'autres activités rémunératrices pour compenser les pertes agricoles se tournent vers la forêt pour la récolte illégale du bois, aggravant ainsi la perte du couvert végétal. Le commerce du bois constitue une source importante de revenus pour bon nombre de riverains.

\section{Le fonctionnement physiologique des arbres}

Les études, même si elles ne permettent pas de conclure pour le moment sur l'impact global des changements appréhendés, ont démontré qu'une augmentation des températures et des concentrations de $\mathrm{CO}_{2}$ pourrait avoir un effet positif sur la croissance et la productivité à long terme de plusieurs essences forestières (GWP 2010). En revanche, la hausse des températures et la diminution de la pluviométrie résultant du changement climatique ont un impact négatif sur la dynamique des ligneux des forêts. Ces phénomènes se traduisent par une mortalité anormalement accrue des arbres (Adjonou et al., 2009). La mortalité des arbres est liée à 
plusieurs facteurs allant de la sécheresse aux attaques nuisibles et aux maladies. Selon FAO (2009), le stress chronique sur de longues périodes affaiblit l'arbre et finit par le tuer. En effet, le stress hydrique dépasse parfois la resistance des arbres entrainant ainsi leur mort. De même, l'augmentation des températures couplées à la diminution de la pluviométrie accroît les incendies des forêts. L'assèchement précoce et prolongé augmente l'intensité des feux de brousse. La Figure 7 montre des pieds d'arbres morts observés dans la forêt.

\section{Le rôle du facteur anthropique sur la forêt Les activités agricoles: l'agriculture et l'élevage}

Les forêts classées sont devenues des espaces convoités par l'agriculture. L'appauvrissement des sols conjugués à la croissance démographique conduit les populations à rechercher de nouvelles terres cultivables. Dans cette recherche, les réserves forestières sont parfois exposées. $\mathrm{La}$ raréfaction des terres cultivables fait que la forêt est souvent perçue comme « une banque de terres » pouvant être utilisée pour l'agriculture lorsque le besoin ou la nécessité s'impose (Sawadogo., 2002). Malgré son statut de réserve classée, on rencontre des champs clandestins d'une superficie non négligeable dans la forêt de Toéssin. L'agriculture constitue un danger pour la survie de cette formation naturelle. En dépit de l'interdiction de faire paître le bétail dans la forêt, l'empreinte de l'élevage est perceptible. Les éleveurs sont confrontés à un manque d'espaces de pâture. En effet, dans un contexte de pression foncière, la mise en place des nouveaux champs se fait au détriment des aires de pâture contraignant ainsi les éleveurs à faire pacager leur troupeau dans la forêt ou à migrer vers d'autres zones. Une pression de pâturage sur la forêt conduit à une destruction du couvert végétal et à une augmentation de la surface de sol nu. Le pacage du bétail dans les zones herbacées et ligneuses détruit le potentiel fourrager de la forêt. Cette destruction est renforcée par les ébranchages d'arbres aux feuilles et aux gousses à grande valeur bromatologique, dus au bétail en saison sèche (Yelkouni, 2004). Par ailleurs, par leur piétinement du sol, les animaux contribuent à accélérer l'érosion hydrique (Sankara, 2011). Les Figures 8 et 9 témoignent de la fréquentation des animaux dans la forêt classée de Toéssin.

\section{Les feux de brousse}

Les enquêtes de terrain ont révélé que durant ces dix dernières années, plusieurs portions de la forêt classée de Toéssin ont été dévastées par des feux incontrôlés (quatre au total). Le feu est un phénomène saisonnier répandu dans les écosystèmes savanicoles. Le comportement du feu est influencé par les variables telles que la topographie, les caractéristiques du combustible et les conditions climatiques du milieu (Guiguindibaye et al., 2013), (Keeley et al., 2012) cité par Doamba et al. 2014. Ainsi, les feux de brousse dont les effets dépendent aussi bien de leurs intensités ainsi que de leur saison d'occurrence, sont considérés comme une perturbation écologique majeure des écosystèmes savanicoles.

Les feux de brousse ont des origines diverses. Ils sont souvent utilisés pour débusquer le gibier dans les environs de la forêt. De plus, pendant la préparation des champs, les feux sont souvent mal contrôlés, de sorte qu'ils peuvent déborder et embraser une partie de la forêt. En vue de protéger la forêt contre les feux, les populations riveraines procédaient à l'ouverture des pare-feux au niveau des zones les plus exposées. Cette mesure de protection n'est plus assurée. Ces feux provoquent des dommages énormes. «Ils détruisent les feuilles et le cambium, brûlent des bois morts et achèvent les arbres malades, diminuant ainsi la récolte potentielle de bois de 
feu ; ils détruisent également le couvert herbacé et soumettent le sol à l'érosion. Au passage du feu, les micro-organismes et les insectes responsables de la décomposition de la matière organique sont tués, ainsi que la faune sauvage » (Yelkouni, 2004 ; Guiguindibaye et al., 2013).

\section{La coupe frauduleuse du bois et le braconnage}

La coupe du bois s'est accentuée avec les besoins de plus en plus grandissants en bois de chauffe. Au Burkina Faso, la principale source d'énergie pour les ménages reste le bois, tant en milieu rural qu'en milieu urbain. La pauvreté croissante entraine aussi une forte exploitation des ressources naturelles des aires protégées. Le bois est devenu un objet de transaction commerciale exacerbant ainsi l'exploitation illégale des ligneux de la forêt classée de Toéssin. En dépit des contrôles imprévisibles du service forestier pour décourager la coupe frauduleuse, les populations riveraines exploitent d'énormes quantités de bois dans la forêt. La coupe du bois (frais) est fréquente et prend des proportions inquiétantes. Les artisans exploitent également de façon frauduleuse les ligneux pour la fabrication de mortier, pilon, chaise et autres ustensiles domestiques. (Nabaloum, 2010). La coupe frauduleuse du bois contribue de manière significative à la dégradation de la forêt. La faune sauvage de la forêt se résumant aux petits gibiers et à l'avifaune fait que la chasse n'est plus très pratiquée par les populations. Néanmoins, certaines personnes continuent de chasser clandestinement les petits mammifères et les oiseaux encore existants dans la forêt.

\section{L'extraction des matériaux de construction}

La quête des matériaux de construction dans la forêt conduit à une modification de la topographie et à une destruction de la végétation. En effet, le creusement de la terre entraine des modifications physiques du sol. Ces modifications sont importantes tant du fait de leur superficie que celui de leur profondeur. En ce qui concerne la végétation, le creusement a occasionné le déracinement de plusieurs individus de Terminalia macroptera et de Terminalia avicennioides. Les arbres, fragilisés par la mise à nue des racines, ne résistent pas très souvent aux grands vents. La «bancotière » n'est plus exploitée, mais les traces sont encore bien visibles. $\mathrm{La}$ reconstitution du couvert végétal dans la zone affectée semble compromise.

Les perspectives de gestion durable de la forêt classée de Toéssin

\section{Les opérations de reboisement}

Les reboisements apparaissent nécessaires pour reconstituer le couvert végétal sur les zones nues. Selon les informations recueillies sur le terrain, il y a plus d'une vingtaine d'années que la forêt n'a bénéficié d'aucune opération de reboisement alors que les ligneux subissent une forte exploitation. Il est plus que jamais nécessaire que la Direction Provinciale en charge de l'Environnement du Passoré en concert avec la population organise de façon périodique des campagnes de reboisement. Au cours des enquêtes, $78 \%$ des personnes de notre échantillon déclarent être prêtes à participer de façon volontaire aux travaux de reboisement de la forêt. Cette motivation est liée aux intérêts socioéconomiques et écologiques qu'elles perçoivent clairement ou qu'elles tirent déjà de la forêt (Nabaloum, 2010).

\section{Lutter contre les empiétements}

La superficie de la forêt varie d'une source à une autre. UICN-PAPACO (2012) mentionne un espace de 490 ha, tandis que d'autres études conduites en 1992 et en 1994 donnent respectivement 701 ha et 774 ha. Il est important de fixer clairement les limites en vue d'empêcher de nouveaux empiètements. Aussi faut-il déguerpir les champs clandestins créés dans la forêt. La présence de ces champs dans la forêt l'expose non seulement aux feux de 
brousse mais aussi à la convoitise d'autres paysans. Les champs existant font aussi l'objet d'agrandissement, ce qui entraine davantage un abattage des arbres. Pour lutter contre les empiètements, il est indispensable également de chercher des solutions contre la dégradation accélérée des sols autour de la forêt. En effet, les différentes sécheresses poussent les paysans à rechercher des terres dans les bas-fonds, par conséquent dans les forêts (Nabaloum, 2010). Dans cette situation, les techniques agricoles d'adaptation aux changements climatiques doivent être pratiquées et maitrisées par tous les agriculteurs. L'agroforesterie doit être associée aux techniques culturales (l'utilisation des variétés améliorées et les techniques d'amendement) et les aménagements (les cordons pierreux). Cela améliorera la qualité des sols, ce qui permettra d'accroitre les rendements agricoles tout en freinant l'extension anarchique et la création des champs dans la forêt.

Impliquer les différents utilisateurs des produits forestiers dans la gestion de la forêt

Les principaux utilisateurs des produits forestiers sont les artisans, les éleveurs, les vendeurs de bois, les femmes (celles qui font la cueillette) (Abegg et al., 2005). Curtis (2014) partage cet avis que les femmes soient en partie les seules à accéder et collecter les fruits, les feuilles, les noix, les graines et les champignons autour des villages en zones rurales au Swaziland sous gestion communale forestière. Cependant, ces différents acteurs ne sont pas impliqués dans la gestion et la préservation des ressources de cette formation naturelle. Les rôles de la forêt dans la satisfaction des besoins fondamentaux des populations riveraines sont multiples et variés. Il est donc nécessaire d'associer les utilisateurs des produits forestiers dans la gestion de la forêt. L'approche participative est indispensable pour la préservation des ressources naturelles de la forêt classée de
Toéssin. Lingani (2011) a mentionné que l'accès aux PFNL pour les femmes à la Sissili au Burkina Faso était 1,2 fois inférieur à celui des hommes. Cela montre que les femmes sont des actrices à intégrer dans le programme d'aménagement de la forêt. L'implication des populations environnantes dans l'aménagement et la gestion des écosystèmes garantirait la viabilité de l'aire protégée (Sarr, 2008). Les objectifs de conservation des aires protégées ne peuvent être atteints que par des actions concertées. Pour Adomou et al. (2017), le cas de la forêt "Bahazoun" au Bénin constitue une caractéristique primordiale mais mérite une surveillance particulière à cause de sa taille très restreinte et son appartenance au Dahomey-Gap.

\section{Conclusion}

La forêt classée de Toéssin bien que bénéficiant de règles de gestion (interdiction de couper le bois vert, de pâturage, de feu), ne reçoit pas des mesures d'accompagnements particulières pour sa gestion. Les forestiers présents ne disposent pas de postes de contrôle et de moyens suffisants pour faire respecter des règles de gestion. Sans minimiser les effets néfastes du climat sur les ressources naturelles, la forte dégradation de la forêt est surtout due aux diverses pressions anthropiques. Les plus menaçantes sont l'agriculture, l'élevage et la coupe frauduleuse $\mathrm{du}$ bois (bois vert). La population riveraine n'est pas organisée en unités forestières locales en appui aux agents forestiers pour sa gestion. Pour faire face aux menaces, des mesures doivent être prises et des perspectives de gestion durable de la forêt définies. Ainsi, l'approche participative de gestion forestière doit être prise en compte, car elle représente une nouvelle restriction d'accès pour les villageois dans la cueillette des PFNL.

Aussi, l'implication des populations environnantes dans l'aménagement et la gestion des écosystèmes garantirait la 
viabilité. L'organisation d'opérations de reboisement et la lutte contre les empiétements dans la forêt contribueraient pour beaucoup à sa sauvegarde.

\section{CONFLITS D'INTERETS}

Les auteurs déclarent qu'ils n'ont pas de conflits d'intérêts

\section{CONTRIBUTIONS DES AUTEURS}

MB a coordonné les travaux de terrain et est le rédacteur principal de cet article. $\mathrm{ZM}$ a élaboré la méthode de recherche et de cartographie et les a appliquées. Il a coordonné les enquêtes de terrain. MN a élaboré le guide d'enquêtes et a contribué aux enquêtes de terrain.

\section{REMERCIEMENTS}

Nos remerciements aux populations de Toéssin et environ, aux services forestiers, de l'élevage et de l'agriculture du département de Samba pour leur coopération lors des enquêtes.

\section{RÉFÉRENCES}

Abegg, Bayala J, Belem OM. 2005. Facteurs socio-économiques influençant la biodiversité ligneuse des parcs agroforestiers de deux villages du plateau central du Burkina Faso. Journal Forestier Suisse, 157: 17-23.

Abdou A. 2010. Variabilité et changements du climat au Sahel: ce que l'observation nous apprend sur la situation actuelle. Grain de Sel, 49 : 13-14.

Adjonou K, Bellefontaine R, Kokou K. 2009. Les forêts claires du parc national OtiKéran au Nord Togo: structure, dynamique et impacts des modifications climatiques récentes, article de recherche, Sécheresse, 20(1): 10.

Adomou CA, Dassou HG, Houenon GHA, Alladayè A, Yedomonhan H. 2017. Comprendre les besoins en ressources végétales des populations riveraines pour une gestion durable de la forêt Bahazoun au Sud-Bénin (Afrique de l'Ouest). Int. J. Biol. Chem. Sci., 11(5): 2058-2070.

Bagaya O. 2013. Approche géomatique des stratégies agricoles d'adaptations aux effets des changements climatiques dans la commune de Dano. Mémoire de Master, Université de Ouagadougou, 93 p.

Belemsobgo U. Adouabou B. 2006. Zones d'importance particulière pour la conservation de la biodiversité au Burkina. In Atlas de la biodiversité de l'Afrique de l'Ouest (Tome II), Thiombiano A, Kampmann D (eds). BIOTA: Burkina Faso.

CIFOR. 2012. Le rôle des forêts et des arbres dans l'adaptation sociale à la variabilité et au changement climatique. Article, $16 \mathrm{p}$.

Curtis RM. 2014. Access and use of forest resources: Evidence from common property forest management in Swaziland. African Journal of Estate and Property Management, 1(1): 008-017. http://hdl.handle.net/10535/9785

Diatta AA, Ndour N, Manga A, Sambou B, Faye CS, Diatta L, Goudiaby A, Mbow C, Dieng SD. 2016. Services écosystémiques du parc agroforestier à Cordyla pinnata (Lepr. ex A. Rich.) Milne-Redh. dans le Sud du Bassin Arachidier (Sénégal). Int. J. Biol. Chem. Sci., 10(6): 2511-2525.

Doamba SWMF, Savadogo P, Nacro HB. 2014. Rôle des feux de savane sur les caractéristiques biogéochimiques des sols en zone soudanienne du Burkina Faso Int. J. Biol. Chem. Sci., 8(2): 777793.

Doamba P. 2012. Impact de l'utilisation des produits forestiers ligneux et non ligneux sur la gestion du parc national d'Arly au Burkina Faso. Master Environnement à l'Institut 2IE Promotion 2010-2012, 58 p. 
FAO. 2009. Études de cas sur l'évolution de la dégradation des forêts. La dégradation des forêts en République Démocratique du Congo. Document de travail, 29 p.

Guiguindibaye M, Belem M, Boussim JI. 2013. Caractéristiques des feux dans un incendie en savane soudanienne au Tchad, Int. J. Biol. Chem. Sci., 7(3): 1147-1156.

GWP/AO// Burkina Faso. 2010. Changement climatique: Inventaire des stratégies d'adaptation des populations locales et échanges d'expériences de bonnes pratiques entre régions du Burkina Faso. Ouvrage, 85 p.

Keeley JE, Bond WJ, Bradstock RA, Pausas JG, Rundel PW. 2012. Fire in Mediterranean Ecosystems. Ecology. Cambridge University Press.

Lingani P. 2011. Appraisal of the participatory forest management program in southern Burkina Faso. Doctoral Thesis $\mathrm{n}^{\circ} 2011$. Faculty of forest sciences. Swedish University of agricultural sciences, 96p.

MECV. 2004. Rapport national sur la gestion durable des forêts au Burkina Faso. 31p.

Nabaloum M. 2010. Impacts des changements climatiques sur la dynamique de la végétation et la production de PFNL. Mémoire de Master II de géographie à l'UFR/SH de l'Université de Ouagadougou, $98 \mathrm{p}$.

Oloukoi J, Mama VJ, Agbo FB. 2006. «Modélisalisation de la dynamique de l'occupation des terres dans le département des collines au Bénin. Télédétection, 6(4) : 305-323.

Sankara TB. 2011. Variabilité climatique et gestion des ressources naturelles : Cas de la forêt classée et réserve partielle de la faune de Gonsé. Mémoire de Maîtrise, Université de Ouagadougou, 109 p.

Sarr SM. 2008. Impacts des pressions anthropiques sur les ressources naturelles du parc national des Deux Balé/Burkina Faso. Institut International d'Ingénierie de l'Eau et de l'Environnement (2iE), Mémoire de Master, 83 p.

Sawadogo M. 2013. Dynamique des espèces ligneuses face à une agriculture itinérante sur brûlis dans la commune de Mangodara: cas des arbres oléagineux. Mémoire de Master, Université de Ouagadougou, $104 \mathrm{p}$.

Sawadogo P. 2002. Pâturages de la forêt classée de Tiogo: diversité végétale, valeur nutritive et utilisations. Mémoire d'ingénierie, Université Polytechnique de Bobo Dioulasso, $149 \mathrm{p}$.

Tarchiani V, Vecchia AD, Pini G, Laminou AM, Toudjani Z, Maman G. 2008. «Approches méthodologiques et outils opérationnels pour la gestion des forêts classées en Afrique de l'Ouest: le cas de Niger ». Sécheresse, 19(4): 261-267, DOI: $10.1684 / \mathrm{sec} .2008 .0148$

UICN-PAPACO. 2012. La gouvernance des aires protégées en Afrique de l'Ouest. Études de cas au Bénin, Burkina Faso et Sénégal. Rapport, Beauvechain (catalogue d'aires d'Afrique de l'Ouest), $164 \mathrm{p}$.

Yelkouni M. 2004. Gestion d'une ressource naturelle et action collective : Le cas de la forêt de Tiogo au Burkina Faso. Thèse de doctorat de troisième cycle de sciences économiques, Université d'Auvergne-Clermont I, 248 p. 